\title{
PROFESSIONAL ACTIVITY OF EMERY-DREIFUSS MUSCULAR DYSTROPHY PATIENTS IN POLAND
}

\author{
AGNIESZKA MADEJ-PILARCZYK \\ Polish Academy of Sciences, Mossakowski Medical Research Centre, Warszawa, Poland \\ Neuromuscular Unit
}

\begin{abstract}
Objectives: Emery-Dreifuss muscular dystrophy (EDMD) is a very rare genetic disorder affecting skeletal and heart muscles. The aim of this study was to identify factors which might influence the ability to work in EDMD patients in Poland. Material and Methods: The study included 24 patients suffering from either of the two EDMD forms: 17 with emerinopathy (EDMD1; EDMD caused by mutations in the emerin gene) and 7 with laminopathy (EDMD2; EDMD caused by the lamin $\mathrm{A} / \mathrm{C}$ gene mutations). After clinical evaluation of EDMD course, study participants were questioned about their education, current and former employment, and disability certificates and pensions. Results: $54 \%$ of the study participants were employed, and $90 \%$ of them had job position corresponding to their education. Undertaking work did not correlate with the level of physical performance or disease complication, but it revealed statistically significant correlation with a higher level of education $(\mathrm{p}=0.015)$. Only $23 \%$ of professionally active patients were employed in a sheltered workplace. Disability certificate was granted to all EDMD2 and to 90\% of EDMD1 patients. All EDMD2 and 50\% of EDMD1 patients received a disability pension, which reflects more severe course of EDMD2. Conclusions: Higher level of education increased the chance of employment, even if significant disability was present. Therefore, I hypothesize that advice on education and job counseling should be applied as early as possible after the diagnosis of EDMD.
\end{abstract}

Key words:

Professional activity, Employment, Laminopathies, Emery-Dreifuss muscular dystrophy

\section{INTRODUCTION}

Employment status of patients suffering from chronic diseases resulting in disability influences their economic status, well-being and quality of life. Several analyses of professional profile of patients suffering from various neuromuscular diseases have been performed. They usually focused on disorders with moderate prevalence, such as facioscapulohumeral muscular dystrophy (FSHD), myotonic dystrophy (MD), and peripheral neuropathy [1,2]. Much less is known about the employment of patients with rare and very rare hereditary muscle diseases, such as Emery-Dreifuss muscular dystrophy (EDMD). The estimated frequency of EDMD is 3:1000000 [3], therefore, the recruitment of the EDMD patients for the study is considered very difficult. On the molecular level, the disease results from the structural and functional defects of nuclear proteins [4].

Two main types of EDMD are known: recessive, X-linked emerinopathy (EDMD1) caused by mutations in the EMD gene encoding emerin, and autosomal dominant laminopathy (EDMD2) caused by mutations in the LMNA gene encoding lamin $\mathrm{A} / \mathrm{C}[5,6]$. Males with EDMD1 present humero-peroneal muscle weakness and wasting, joint contractures, spine rigidity, and cardiomyopathy with conduction abnormalities. Muscle symptoms usually appear in the first decade of life and precede cardiac symptoms which

Received: September 30, 2013. Accepted: January 29, 2014

Corresponding author: A. Madej-Pilarczyk, Neuromuscular Unit, Mossakowski Medical Research Centre, Polish Academy of Sciences, Pawińskiego 5, $02-106$ Warszawa, Poland (e-mail: agamadpil@gmail.com). 
are typically detected in the second or third decade of life. The muscle system of female carriers of the EDMD1 mutation is not affected, but in the fourth or fifth decade of life $20 \%$ of them have symptomatic cardiac disease, as EDMD1 men [7]. Clinical manifestation of EDMD2 is similar in both sexes, but, in comparison to EDMD1, muscle symptoms in EDMD2 may occur earlier or later than cardiac disease. Patients with EDMD2 have greater probability to lose their ability to walk independently than patients with EDMD1, but in both types of EDMD patients with less severely affected skeletal muscle system can be seen. In addition, significant intra- and inter-familial variation in clinical symptoms of EDMD is observed [5-7]. Cardiac symptoms can be life-threatening in both types of EDMD. In EDMD1, a sudden cardiac death is usually a result of a complete atrioventricular block, and might be prevented by pacemaker implantation. In EDMD2, systolic dysfunction of the heart, left ventricle dilation, and life-threatening ventricle arrhythmia predominate; this requires implantation of cardioverter-defibrillator and, in the end-stage cardiac insufficiency, heart transplantation $[8,9]$.

Patients with both types of EDMD are at a higher risk of complications, which might further worsen the level of physical performance. Arrhythmia and heart enlargement predispose to ischemic strokes, while fast-progressing cardiomyopathy might lead to life-threatening cardiac and respiratory failure [10].

Therefore, both types of EDMD might lead to disability and might strongly affect patient's professional career. Notably, there are no reports regarding the employment status of EDMD sufferers. In this work, I analyze the professional activity of a large group of Polish EDMD patients in relation to the level of their education and disease-associated factors, such as the level of their physical performance, complications and cardiology interventions. The aim of this study was to identify factors which might influence the ability to work in EDMD patients in Poland.

\section{MATERIAL AND METHODS}

The study group consisted of 24 patients diagnosed with EDMD, remaining under the care of the Neuromuscular Unit, Mossakowski Medical Research Centre, who have agreed to answer some questions on their education and professional activity. All patients expressed their written informed consent for participation in the study. In accordance with the rules regarding questionnaire-based non-interventional studies analyzing nonsensitive data, the Bioethical Committee was informed on the current questionnaire survey. The obtained data was analyzed anonymously, with no possibility to identify the patients.

Seventeen of the 24 patients ( 3 female carriers, 14 males) suffered from EDMD1, and 7 (3 females, 4 males) from EDMD2. The mean age of all of the EDMD patients was $34.4 \pm 11.4$ years (range: $18-56$ years). Only one patient (4.2\%) was less than 20 years old, $7(29.2 \%)$ were 20 30 years old, 7 (29.2\%) were 31-40 years old, 8 (33.2\%) were $41-50$ years old, and 1 (4.2\%) was older than 50 . The analyzed patients were divided into 3 groups according to the severity of EDMD (Table 1). Treatment included, but was not limited to, a specialist cardiology intervention: cardiac devices were implanted in 19 (79.1\%) EDMD patients: 13 of the 14 EDMD1 patients, 1 of the 3 EDMD1 carriers and 2 of the 7 EDMD2 patients have pacemakers, while 3 of the 5 EDMD2 patients have cardioverter defibrillators. Disease complications/injury/concomitant disease occurred in 10 (41.6\%) patients. Types of events were also described in Table 1.

I have analyzed attitude to work (undertook/did not undertake) of the EDMD patients in relation to education and the severity of the underlying disease (the level of physical performance depending on EDMD severity, cardiology interventions, disease complications), then the association of certificates of disability granted with the course of the EDMD. The relationships between the above mentioned parameters and EDMD course/level of 


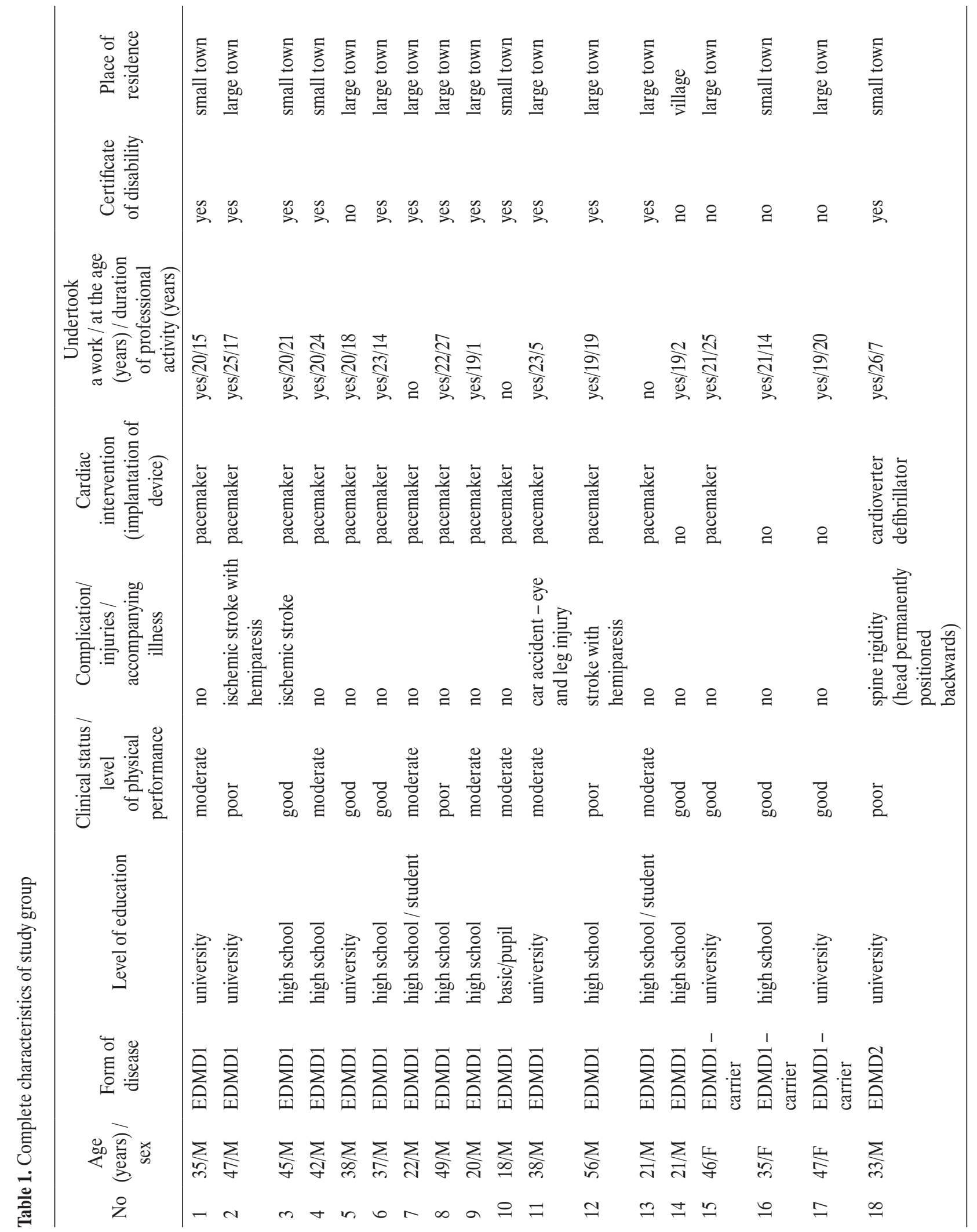




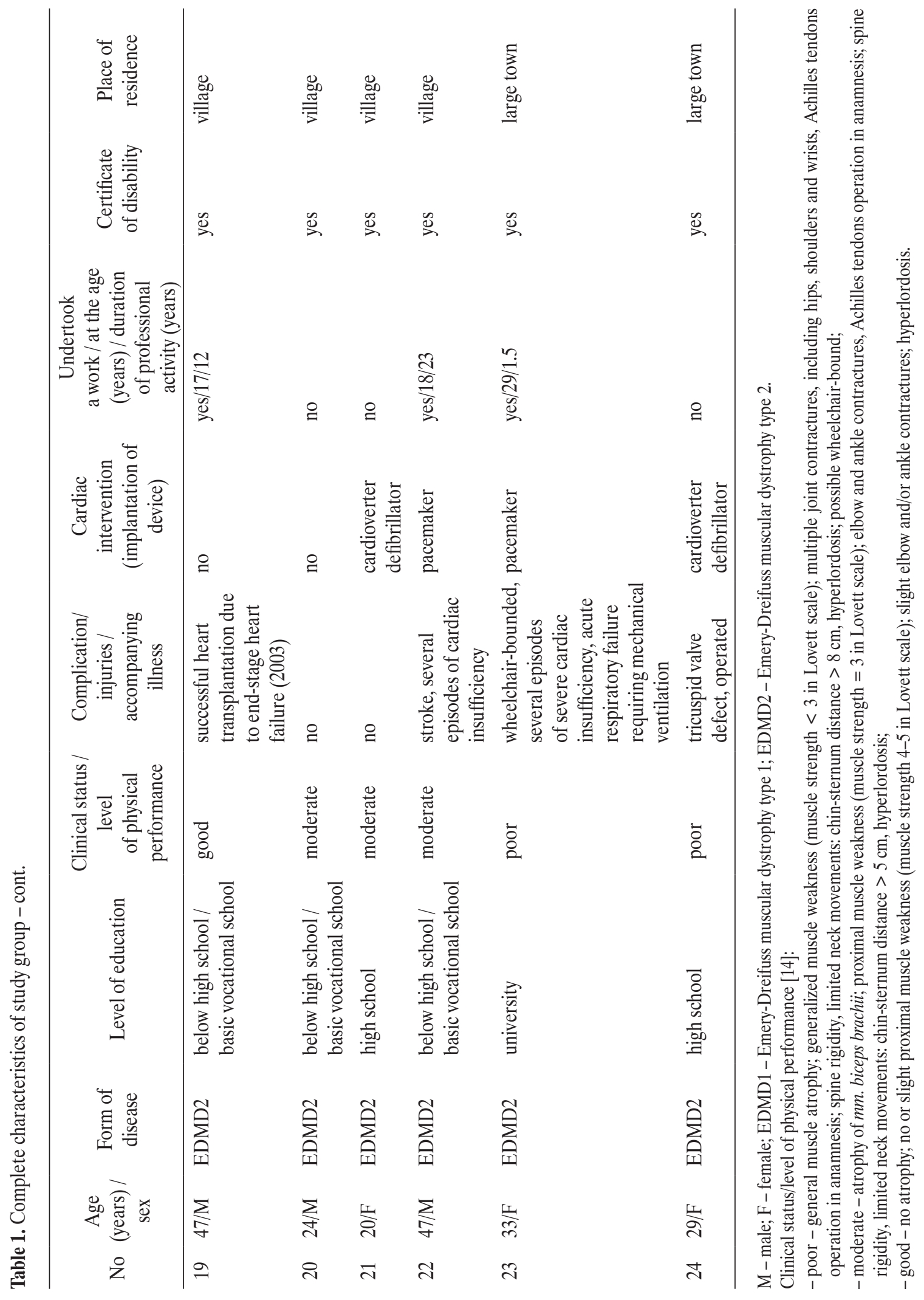


education were analyzed using chi-squared test, while the duration of professional activity with EDMD severity/ level of education was analyzed by Spearman's rank correlation coefficient (rho). The $\mathrm{p}<0.05$ value was considered to be statistically significant. Patients with EDMD1 and EDMD2 were compared in the descriptive way in terms of their certificates of disability and disability pensions.

\section{RESULTS}

\section{Education}

University-level education was earned by 7 (29.17\%) EDMD patients, $10(41.67 \%)$ graduated from high schools, $3(12.5 \%)$ finished their education at below high school-levels, and 4 (16.67\%) patients were still students/ pupils. The level of education did not differ in EDMD1 and EDMD2 patients $\left(\mathrm{Chi}^{2}(4)=6.67 ; \mathrm{p}=0.117\right)$. Five of 7 patients who earned university-level education lived in large towns and the remaining 2 patients - in small towns.

Education level did not correlate with the level of physical performance (rho $=-0.14 ; \mathrm{p}=0.520$ ), cardiology intervention $\left(\mathrm{Chi}^{2}(4)=5.90 ; \mathrm{p}=0.169\right)$ or possible disease complications $\left(\mathrm{Chi}^{2}(4)=4.47 ; \mathrm{p}=0.275\right)$.

\section{Professional activity}

Six of the 24 of the analyzed EDMD patients (25\%) have never undertaken work. The age at the first employment depended on the level of education and was compatible with the length of educational path. Patients with their education below the high school-level usually took their first jobs at the age of 17-18 years, with completed high school education - at the age of 1822 years, and with university-level education - at the age of 21-29 years. Undertaking work did not correlate with the level of physical performance $\left(\mathrm{Chi}^{2}(2)=5.64\right.$; $\mathrm{p}=0.056)$, cardiology intervention $\left(\mathrm{Chi}^{2}(1)=0.00\right.$; $\mathrm{p}>0.999)$ or disease complications $\left(\operatorname{Chi}^{2}(1)=0.53\right.$; $\mathrm{p}=0.465)$, but it revealed statistically significant correlation with higher level of education $\left(\mathrm{Chi}^{2}(3)=8.55\right.$; $\mathrm{p}=0.015)$.

Thirteen (54\%) of the EDMD patients were professionally active at the time of the survey; 12 of them (92\%) worked in professions concordant with their education. Seven of the 8 patients with university-level education (3 EDMD1, 2 EDMD1 carriers, 2 EDMD2) were employed regardless of moderate and high level of their disability. Non-working group consisted of $11(46 \%)$ patients, including 3 still at school. Among the 21 patients who completed their education, 3 (14.3\%) had never worked. Professional positions of the EDMD patients were:

- a clerk, 6 patients (3 with university-level education, 3 with high school-level education);

- an IT specialist, 2 patients (both with university-level education);

- a manual worker, 2 patients (1 with high school-level education, 1 with below high school-level education);

- a car driver, 1 (high school-level education);

- a psychologist, 1 (university-level education);

- a librarian, 1 (university-level education).

Professionally active patients were usually full-time employees. One person was a full-time employee and, in addition, had a part-time job. Another patient was a full-time employee and ran his own business. Three out of the $13(23 \%)$ professionally active EDMD patients worked in a sheltered workplace. None of the 5 people who had been working in the past, but were then not employed, had ever worked in a sheltered workplace.

The mean duration of professional activity was $14.7 \pm 8.4$ years (range: $1-27$ years). The duration of their working career depended rather on the patient's age at the time of survey, while it was not correlated with the level of physical performance, cardiology intervention, complications or education ( $r$ ho $=0.05 ; 0.22 ;-0.17 ;-0.17$, respectively). 


\section{Certificate of disability}

The majority of the EDMD patients (17 of 24; 71\%) had a certificate of disability. Only five (20.8\%) study participants (3 female EDMD1 carriers and 2 EDMD1 male patients) had no such certificate. The mean age at which the first certificate of disability was issued was $19 \pm 9.1$ years (age range: 6-38 years) and it depended on the level of physical performance (statistically significant correlation: $\left.\mathrm{Chi}^{2}(2)=6.34 ; \mathrm{p}=0.035\right)$ and on complications $\left(\operatorname{Chi}^{2}(1)=3.89 ; p=0.049\right)$, while it was not dependent on cardiology intervention $\left(\mathrm{Chi}^{2}(1)=2.69 ; \mathrm{p}=0.101\right)$.

In fact, patients with predominant skeletal muscle symptoms received their first certificate of disability at the age of approximately 20 years, while patients with predominant cardiac symptoms received the certificate at the age of over 30 years. EDMD1 patients with pronounced skeletal muscle symptoms received their certificates earlier than the EDMD1 patients with mild skeletal muscle symptoms and the EDMD2 patients with cardiology symptoms which appeared earlier than muscle symptoms. Two EDMD1 and 1 EDMD2 patients had the certificate of disability issued before they were 10 years old; it was due to the high awareness of the disease in their families in which other members suffered from EDMD as well as early genetic confirmation of clinical diagnosis of EDMD, and, in one case of the EDMD1 patient, due to very severe skeletal muscle symptoms that resulted in wheel-chair bounding at the age of 9 years.

Six of the $7(85.71 \%)$ EDMD2 patients had the permanent or temporary first disability group, which is consistent with the severe course of EDMD associated with laminopathy, affecting both skeletal muscles and the heart. The first disability group was held in 3 EDMD1 patients and 2 of them had an additional deteriorating factor - ischemic stroke.

\section{Disability pension}

Disability pension was granted to all of the 7 EDMD2 patients (100\%), to 7 of the $14(50 \%)$ EDMD1 patients and 2 additional patients (students) from the latter group had a social (welfare) pension. No EDMD1 female carrier requested a disability pension, despite the symptomatic course of their disease with cardiac manifestation, requiring a pacemaker implantation. Permanent disability pension was granted to 3 out of the 14 EDMD1 patients, including 2 oldest patients: the first one received it after 3 cerebral strokes, and the second one received it due to a severe muscle hypotonia.

Permanent disability pension was also granted to 3 out of 7 EDMD2 patients, including a wheelchair-bound female with severely affected muscles and another female with life-threatening cardiac arrhythmia with implanted cardioverter defibrillator. Notably, the EDMD2 patient with progressive muscle atrophy and weakness, who had heart transplantation 17 years ago, had not been granted a permanent disability pension.

\section{DISCUSSION}

Although Emery-Dreifuss muscular dystrophy results in a significant health burden, both due to its influence on general physical condition and to serious heart consequences, $54 \%$ of the tested EDMD patients were employed at the time of the survey. Until now, there have been no reports regarding professional activity of EDMD patients. In the large analysis of the employment status of patients with various neuromuscular diseases described by Fowler et al., the percentages of employed patients was only $40 \%$, and in patients with myotonic dystrophy (MD) and limb-girdle muscular dystrophy (LGMD) it was even lower [11]. In the analysis of Wevers et al., the percentage of patients with facioscapulohumeral muscular dystrophy (FSHD) who had a job was 44\% [2], while in the report of Minis et al., the percentage of working myotonic muscular dystrophy patients was $47.8 \%$, facioscapulohumeral dystrophy patients $-70.3 \%$, and in the case of hereditary motor and sensory neuropathy 
type I it was $63.7 \%$ [1]. Importantly, patients with neuromuscular diseases want to work: the percentage of patients who had never taken a job was very low in our analysis and in the analysis of Fowler et al., only $14.4 \%$ and $10 \%$, respectively [12].

In our analysis, the percentage of the employed EDMD patients is slightly higher than in the majority of patients with other neuromuscular diseases, i.e., FSHD, LGMD and MD, described by other authors $[1,2,11]$. Obviously, this is not due to the less severe course of this disease, but it might be a result of a relatively low mean age of our study participants compared to the other studied groups. Since the symptoms of neuromuscular disorders usually increase with age, younger age is one of the most important factors increasing the chance of employment [12].

Employment status of EDMD1 female carriers seems to be associated mainly with disease-independent factors, e.g., availability of work and family situation, as these patients have no muscle symptoms and their cardiac disease (if present) is quite well controlled by pacemaker implantation and pharmacotherapy.

The major barrier for employment in the analyzed group is probably less dependent on the level of physical performance, cardiology interventions or disease complications, but rather on the lack of education, although the sample size is too small and the study group is not enough homogenous to reveal unquestionable statistical differences. Higher level of education gives a better chance to get a job which does not require heavy manual work and might be fitted to the restricted physical performance of the EDMD patients, and allows to retain professional activity for a longer period even in the case of severe disability (remote work). Therefore, education is another significant factor for people with neuromuscular diseases to enter/ remain on labor market. Job counseling is crucial for the choice of appropriate education path, corresponding with health restrictions, increasing a chance to find a satisfying position $[1,10,12]$. Indeed, almost all of the patients from our group who completed university-level education were professionally active. The majority of patients ( 5 of 7 ) who had earned university-level education lived in large towns. It is not surprising that their place of residence, i.e., rather in a town than in a village, is yet another factor increasing their chance to get higher education through the accessibility of university-level schools, allowing to get a job compatible with their skills.

The duration of the professional career was dependent on patient's age, reaching up to 18 years in 30-40 years old patients and up to 27 years in patients $>40$ years old. This observation is similar to that described by Wevers et al., who observed that the average working career length was 15 and 30 years for women and for men with FSHD, respectively [2]. In Poland, only several patients were employed in sheltered workplaces for disabled, although such workplaces allow prolonging the period of professional activity, especially for people with lower qualifications. Similarly, low percentage of patients (approximately 10\%) with FSHD having workplace adjustments for diseaserelated handicaps was reported by Wevers et al. [2].

Since the disease course in EDMD2 is generally more severe than in EDMD1 and patients with EDMD2 more often lose ability to walk independently and have lifethreatening cardiac symptoms which require a device implantation or heart transplantation, it is reflected in the number of the issued certificates of disability (100\%) and disability groups (usually I, often permanent). The level of disability in the EDMD1 patients is influenced by age and additional medical conditions, very often associated with the underlying disease (e.g. stroke secondary to dilated cardiomyopathy or cardiac arrhythmia). The percentage of patients receiving a disability pension is also higher in EDMD2 group, reaching 100\%, while, in EDMD1, it is $50 \%$. In the longitudinal analysis of Bostrom et al. regarding the impact of sickness in 44 adult patients with various types of muscular dystrophy, the percentage of disability pension ranged from 36 to $55 \%$, depending on 
age, which is similar to our observation in the EDMD1 patients, but not in the EDMD2 patients [13].

In summary, regardless of their disability, the EDMD patients are willing to work. Since, higher level of education seems to increase the chance of employment of the EDMD patients, it would be appropriate to advise on education and to introduce job counseling as early as possible after the diagnosis of the disease. This might result in a better quality of life, social and economic status of the EDMD patients.

\section{REFERENCES}

1. Minis MA, Kalkman JS, Akkermans RP, Engels JA, Huijbregts PA, Bleijenberg G, et al. Employment status of patients with neuromuscular diseases in relation to personal factors, fatigue and health status: A secondary analysis. J Rehabil Med. 2010;42(1):60-5.

2. Wevers CW, Brouwer OF, Padberg GW, Nijboer ID. Job perspectives in facioscapulohumeral muscular dystrophy. Disabil Rehabil. 1993;15(1):24-8, http://dx.doi. org/10.3109/09638289309165865.

3. Orphanet Report Series [Internet] [updated 2014 Jan 19; cited 2014 Jan 20]. Available from: http:/www.orpha.net/orphacom/cahiers/docs/GB/Prevalence_of_rare_diseases_by_ alphabetical_list.pdf.

4. Broers JL, Ramaekers FC, Bonne G, Yaou RB, Hutchison CJ. Nuclear lamins: laminopathies and their role in premature ageing. Physiol Rev. 2006;86(3):967-1008, http:// dx.doi.org/10.1152/physrev.00047.2005.

5. Bione S, Maestrini E, Rivella S, Mancini M, Regis S, Romeo $\mathrm{G}$, et al. Identification of a novel X-linked gene responsible for Emery-Dreifuss muscular dystrophy. Nat Genet. 1994;8(4):323-7, http://dx.doi.org/10.1038/ng1294-323.

6. Bonne G, di Barletta MR, Varnous S, Bécane HM, Hammouda EH, Merlini L, et al. Mutations in the gene encoding lamin $\mathrm{A} / \mathrm{C}$ cause autosomal dominant Emery-Dreifuss muscular dystrophy. Nat Genet. 1999;21(3):285-8, http://dx.doi. org/10.1038/6799.
7. Emery AE. Emery-Dreifuss muscular dystrophy - A 40 year retrospective. Neuromuscul Disord. 2000;10(4-5):228-32.

8. Sanna T, Dello Russo A, Toniolo D, Vytopil M, Pelargonio G, de Martino G, et al. Cardiac features of Emery-Dreifuss muscular dystrophy caused by lamin $\mathrm{A} / \mathrm{C}$ gene mutations. Eur Heart J. 2003;24:2227-36, http://dx.doi.org/10.1016/ j.ehj.2003.09.020.

9. Taylor MR, Fain PR, Sinagra G, Robinson ML, Robertson $\mathrm{AD}$, Carniel E, et al. Familial dilated cardiomyopathy registry research group: Natural history of dilated cardiomyopathy due to lamin $\mathrm{A} / \mathrm{C}$ gene mutations. J Am Coll Cardiol. 2003;41:771-80, http://dx.doi.org/10.1016/S07351097(03)00887-8.

10. Boriani G, Gallina M, Merlini L, Bonne G, Toniolo D, Amati S, et al. Clinical relevance of atrial fibrillation/flutter, stroke, pacemaker implant, and heart failure in Emery-Dreifuss muscular dystrophy: A long-term longitudinal study. Stroke. 2003;34(4):901-8, http://dx.doi.org/10.1161/01. STR.0000064322.47667.49.

11. Fowler WM Jr, Abresch RT, Koch TR, Brewer ML, Bowden RK, Wanlass RL. Employment profiles in neuromuscular diseases. Am J Phys Med Rehabil. 1997;76(1):26-37.

12. Minis MA, Heerkens Y, Engels J, Oostendorp R, van Engelen B. Classification of employment factors according to the International Classification of Functioning, Disability and Health in patients with neuromuscular diseases: A systematic review. Disabil Rehabil. 2009;31(26):2150-63, http:// dx.doi.org/10.3109/09638280902951838.

13. Boström K, Nätterlund BS, Ahlström G. Sickness impact in people with muscular dystrophy: A longitudinal study over 10 years. Clin Rehabil. 2005;6:686-94, http://dx.doi. org/10.1191/0269215505cr866oa.

14. Bonne G, Leturcq F, Ben Yaou R. Emery-Dreifuss muscular dystrophy. In: Pagon RA, Adam MP, Bird TD, Dolan CR, Fong CT, Stephens K, editors. GeneReviews ${ }^{\mathrm{TM}}$ [Internet]. Seattle (WA): University of Washington; 1993-2013 [updated 2013 Jan 17; cited 2014 Jan 20]. Available from: http:// www.genereviews.org.

This work is available in Open Access model and licensed under a Creative Commons Attribution-NonCommercial 3.0 Poland License - http://creativecommons.org/ licenses/by-nc/3.0/pl/deed.en. 